\title{
Placebo in new Covid-19 vaccine trials: data quality prioritised over participants' rights
}

\author{
SANDHYA SRINIVASAN
}

\begin{abstract}
The WHO Ad Hoc Expert Group on the Next Steps for Covid-19 Vaccine Evaluation makes recommendations on the use of placebo controlled trials in ongoing and future Covid-19 vaccine research. These recommendations unequivocally prioritise data quality over participants' rights and safety. Participants in trials of vaccines which have received emergency use listing or authorisation would be refused available vaccines. Placebocontrolled trials that would be impossible to conduct in rich countries would be permitted in poor countries. If these suggestions are implemented, the major beneficiary will be the vaccine industry.
\end{abstract}

Key words: Covid-19 vaccine trials, ethics of placebo controlled trials, vaccine access, ethics dumping, trial participants'rights.

The race for Covid-19 vaccines has been crowded and chaotic. There are 99 candidate vaccines in 273 human clinical trials as of March 21, 2021. Thirteen vaccines have received emergency use authorisation from one or more regulatory authorities on the basis of interim data of varying quality. The safety and efficacy of all vaccines must be established through ethically and scientifically conducted research, and the guidance of scientists and ethicists is needed in this.

However, the recommendations of the WHO Ad Hoc Expert Group on the Next Steps for Covid-19 Vaccine Evaluation (1) unequivocally prioritise data quality over participants' rights and safety. Participants in trials of vaccines which have received emergency use listing or authorisation would be refused available vaccines. Placebo-controlled trials that would

Author: Sandhya Srinivasan (sandhya199@gmail.com), Independent Researcher and Journalist, Seadoll, 54 Chimbai Road, Bandra (W), Mumbai 400050 INDIA.

To cite: Srinivasan S. Placebo in new Covid-19 vaccine trials: data quality prioritised over participants' rights. Indian J Med Ethics. 2021 Apr-Jun; 6(2) NS: 103-105. DOI: 10.20529/IJME.2021.025.

Published online first on March 31, 2021.

Manuscript Editors: Ruth Macklin, Amar Jesani.

Peer reviewer: An anonymous peer reviewer.

(c) Indian Journal of Medical Ethics 2021 be impossible to conduct in rich countries would be permitted in poor countries. If these suggestions are implemented, the major beneficiary will be the vaccine industry.

The Ad Hoc Expert Group - which includes the director general of the Indian Council of Medical Research and the chief scientist of the World Health Organization, giving the recommendations the stamp of approval of these two organisations makes recommendations to be implemented "while vaccine supplies are limited, available vaccines are still investigational or public health recommendations to use those vaccines have not been made", in order to get the best quality of data in ongoing placebo-controlled trials of Covid-19 vaccines. It also makes recommendations regarding data collection for approved vaccines and for future Covid-19 vaccine trials.

No ethics-based argument is given in support of these suggestions. Nor is there any reference to the factors determining access to Covid-19 vaccines across the world, which have a bearing on the ethics of this research.

\section{Background of discussion on placebos}

Standards for the use of a placebo in clinical trials are given in the ethical guidelines of the World Medical Association's (WMA) Declaration of Helsinki (2) and the Council for International Organisations of Medical Sciences (3). The use of a placebo is largely restricted to conditions for which there is no proven/efficacious intervention.

When an effective drug or intervention exists, testing a new drug or other intervention against a placebo could put research participants at risk of harm. In such circumstances, the use of placebo is acceptable only if it is methodologically necessary and will not cause severe or irreversible harm to the participant (2: para 33).

As Covid-19 is a potentially fatal disease, once a vaccine against the disease is proven efficacious, testing new Covid-19 vaccines against a placebo would be unethical. Participants receiving the placebo would be exposed to a disease that, in a small but significant number of people, results in death or severe damage to health.

In 2014, a WHO Expert Panel Consultation argued that the 
WMA and CIOMS guidelines did not specifically discuss the use of placebo in vaccine trials, and proposed that placebo would be ethical in certain situations (4). In December 2020, as emergency use authorisations started being granted for Covid-19 vaccines, a WHO Policy Brief was published on ethical considerations for placebo-controlled trials for Covid-19 vaccines under emergency use authorisation (5).

The WHO Ad Hoc Expert Group does not refer to any of these documents.

\section{Emergency use authorisation and placebo}

The question is whether emergency use authorisation (EUA) constitutes a proven or efficacious intervention as understood in ethical guidelines. The WHO's Policy Brief on the use of placebos in trials of Covid-19 vaccines holds that interventions under emergency use may not necessarily satisfy this standard. Approval based on interim analysis of data may not necessarily meet trial-stopping rules which would require unblinding and offering the proven effective intervention to the control group. Continuance of placebo-controlled trials of vaccines under emergency use authorisation is scientifically necessary and does not violate current ethical guidance documents (5).

The first recommendation of the Ad Hoc Expert Group is in line with these recommendations. Ongoing placebo-controlled trials of vaccines that have received EUA should go on as planned. Long-term follow up maintaining the placebo control arm is necessary to collect information such as how long immunity lasts, and whether waning protection could lead to vaccine-enhanced disease.

The problem is that the Ad Hoc Expert Group also suggests that it is "ethically appropriate" for trial sponsors not to offer their vaccine under EUA to those in the placebo group of their trial. It suggests that it is appropriate to refuse to tell participants who wish to take another Covid-19 vaccine whether they had received the placebo or vaccine.

In contrast, the Policy Brief advises that as Covid-19 vaccines become available to priority groups, trial participants from these priority groups should be given the option of being unblinded and taking the EUA vaccine. Its position on whether participants outside these priority groups should be given the same option is unclear, though it underlines that the right of participants to withdraw from a trial must be respected (5).

\section{Participants' understanding of emergency use}

Vaccines with EUA have been approved with two months of follow-up after the second dose, with the understanding that all but the rare side effects should have been identified. Follow-up for long-term safety and efficacy could arguably be conducted by other methods, including a crossover of placebo and intervention groups, and surveillance in the community for adverse events following immunisation, which is in any case critical for new vaccines.
It is also relevant that at least one vaccine manufacturer has done a complete analysis of the data, and reported an efficacy of more than $90 \%$. And at least two manufacturers are now offering the vaccine to those in the placebo control, possibly because participants protested and even started dropping out of trials and being lost to follow-up (6).

The vaccines with EUA have been distributed to more than 180 million people as of February 2021. In such a situation, the distinction between EUA and full approval may not be significant to the hundreds of thousands of people worldwide who are participating in clinical trials of vaccines against Covid-19. Those who gave their informed consent would have joined these trials out of a sense of altruism. The informed consent forms they signed are unlikely to have stated that any request for unblinding would be refused. Participants now being asked to agree to wait for up to two years before getting the vaccine (7) are naturally angry. If they are prevented from taking a vaccine that is being given to millions of people -- and that they would be entitled to outside the trial only because it is under EUA their own wellbeing is clearly secondary to the needs of the trial.

International guidelines state clearly that the goal of generating new knowledge "can never take precedence over the rights and interests of individual research subjects." (2: para 8). The Ad Hoc Expert Group's recommendation that researchers may refuse to inform a participant whether s/he received the placebo or investigational vaccine in order to take the vaccine elsewhere could also be viewed as recommending a violation of the participant's right to withdraw from the study at any time, without fear of reprisal (2: para 26).

\section{Ethics dumping}

Second, the Ad Hoc Expert Group proposes that even after some vaccines get full approval, new vaccines should be tested in placebo-controlled trials. The Group gives no ethical justification for this suggestion that would violate international research ethics guidelines $(2,3)$. It suggests that such trials could be conducted in countries with "limited or no access to a known effective vaccine", following the "standard of care of the locality". Such trials would be ethical if the vaccines tested are potentially relevant to the country where they are tested and if once approved, the "community shares in the benefit".

Such research would exploit inequities in vaccine access across countries. Availability of Covid-19 vaccines has been restricted by the vaccine industry that prevents free use of technologies for a public good, in a pandemic, and by rich countries which can make advance purchase agreements for more vaccines than they need (8). The expert group's suggestion is vague as to what may or may not be tested in such placebo-controlled trials - as long as they are of "potential relevance" to the country where the trial will be carried out - and vague about how the country should benefit, as long as it would "share in the benefit". One can see how such trials will be conducted in 
order to obtain data that the vaccine industry cannot obtain in rich countries. The results of these trials will be used towards obtaining approval in those rich countries.

The WHO Expert Panel on placebos in vaccine trials proposes specific situations in which placebo controls may be used when there is already an approved vaccine. Some of its recommendations are clearly debatable and seek a way around problems such as the high prices of vaccines, rather than challenge the structure that permits industry to charge such prices. Still, the Panel proposes that vaccines being tested must address a local health need, and not simply be applicable to the community. Proven effective vaccines must be made available to the community (4). The Ad Hoc Expert Group, on the other hand, looks for how to get the best data on a vaccine, and not what the local community needs.

The Ad Hoc Expert Group argues for placebo-controlled trials even after an effective Covid-19 vaccine is approved because they are more efficient than other trial designs, such as randomised, noninferiority trials. This is essentially an argument of expediency and cost. Placebo-controlled trials need fewer participants, give clear results faster, are therefore cheaper to conduct, and will be useful for the vaccine industry.

\section{Unethical use of placebo in clinical trials - a rich history}

The Ad Hoc Expert Group's recommendations would actually open a door that was shut decades ago, at least officially, on the differential use of placebos in rich and poor countries.

There is a rich history of placebo-controlled trials that would be viewed as unethical in rich countries being conducted in poor countries, on the argument that poor countries would also benefit, or simply because it is easier, cheaper and faster (9). It was criticism of such practices that led to amendments in international as well as national guidelines restricting the use of placebo.

Of course, it is a matter of shame that regulatory bodies have not enforced these guidelines, and ethics bodies have not made enough of a fuss about studies using a placebo when an effective drug or intervention exists. For example, in India, trials funded by the US government and international funding agencies compared inexpensive cervical cancer screening techniques with no screening, instead of against a cytological smear (10). Such a trial would not be permitted in the US. And in the middle of the pandemic, 40,000 people in Guinea Bissau were enrolled in a randomised controlled trial of home-made masks to prevent Covid-19 transmission (11). The trial, run by a university in Denmark, commenced after the Danish government mandated the use of masks in public.

The Ad Hoc Expert Group's recommendations will encourage the proliferation of such unethical research.

While ethics demands that research in a pandemic should strive to obtain the best quality of data, randomised placebocontrolled trials may not always be the ethical choice. Other methods of collecting data, such as equivalence trials, noninferiority trials, crossover trials, observational studies, and long-term follow-up of vaccinees for immunogenicity and safety contribute to the body of evidence on the vaccine. Long-term follow-up of trial participants should be part of all vaccine studies. Information from vaccine adverse events surveillance of the immunisation programme is an ethical imperative, rather than the unrestricted use of placebocontrolled trials to meet the needs of the vaccine industry.

\section{References}

1. WHO Ad Hoc Expert Group on the Next Steps for Covid-19 Vaccine Evaluation. Placebo-Controlled Trials of Covid-19 Vaccines - Why We Still Need Them. N Engl J Med. 2021 Jan 14; 384(2):e2. Doi: 10.1056/ NEJMp2033538. Epub 2020 Dec 2

2. World Medical Association. WMA Declaration of Helsinki - Ethical principles for medical research involving human subjects. Fortaleza, Brazil:WMA; 2013 Oct [cited 2021 Feb 20]. Available from: https:// www.wma.net/policies-post/wma-declaration-of-helsinki-ethicalprinciples-for-medical-research-involving-human-subjects/

3. Council for International Organisations of Medical Sciences, World Health Organization. International Ethical Guidelines for Health Research Involving Humans. Geneva: CIOMS; 2016 [cited 2021 Feb 20]. Available from: https://cioms.ch/publications/product/internationalethical-guidelines-for-health-related-research-involving-humans

4. Rid A, Saxena A, Baqui AH, Bhan A, Bines J, Bouesseau MC, et al. Placebo use in vaccine trials: recommendations of a WHO expert panel. Vaccine. 2014 Aug 20; 32(37):4708-12. Doi: 10.1016/j.vaccine. 2014.04.022. Epub 2014 Apr 25.

5. World Health Organization. Emergency use designation of COVID-19 candidate vaccines: Ethical considerations for current and future COVID-19 placebo controlled vaccine trials and trial unblinding. Policy brief. 2020 Dec 18[cited 2021 Feb 20]. Available from: https:// www.who.int/publications/i/item/emergency-use-designation-ofcovid-19-candidate-vaccines-ethical-considerations-for-current-andfuture-covid-19-placebo-controlled-vaccine-trials-and-trialunblinding

6. Peres J.Vaccine trial participants who received placebo now hop the line for the real thing from Pfizer, Moderna. Chicago Tribune. 2021 Jan 14[cited $2021 \quad$ Feb 20]. Available from: https:// www.chicagotribune.com/coronavirus/ct-life-covid-vaccine-placebopfizer-moderna-janssen-trial-20210114r6vzmohbs5ed5gstqwuxdhtria-story.html

7. Zimmer C, Weiland M. Many trial volunteers got placebo vaccines. Do they now deserve the real ones? New York Times. 2020 Dec 2[cited 2021 Feb 20]. Available from: https://www.nytimes.com/2020/12/02/ health/covid-vaccine-placebo-group.html

8. Human Rights Watch. "Whoever finds the vaccine must share it". Strengthening human rights and transparency around Covid-19 vaccines. 2020 Oct [cited $2021 \mathrm{Feb}$ 20]. Available from: https:// www.hrw.org/sites/default/files/media_2020/10/ globalvaccine1020_web.pdf

9. Lurie P, Wolfe S. Unethical trials of interventions to reduce perinatal transmission of the Human Immunodeficiency Virus in developing countries. N Engl J Med. 1997 Sep 18; 337 (12): 853-56. Doi: 10.1056/ NEJM199709183371212.

10. Schroeder D, Cook Lucas J, Fenet S, Hirsch F, eds. "Ethics Dumping" -Paradigmatic Case Studies, a report for TRUST; 2016 [cited 2021 Feb 20]. Available from: http://trust-project.eu/wp-content/uploads/ 2016/03/TRUST-664771-Paradigmatic-Case-Studies-WP1-Final.pdf

11. Peeples L. Face masks: what the data say. Nature. 2020 Oct 6 [cited 2021 Feb 20]. Available from: https://www.nature.com/articles/ d41586-020-02801-8?sf241112751=1 